\title{
High peripheral and axial bone densities in a postmenopausal woman with untreated hypoparathyroidism
}

\author{
B. Orr-Walker ${ }^{1}$, R. Harris ${ }^{2}$, I.M. Holdaway' ${ }^{1}$ G. Foote ${ }^{3}$ and I.R. Reid ${ }^{1}$ \\ ${ }^{1}$ Department of Medicine, University of Auckland, ${ }^{2}$ Department of Geriatrics, Auckland Hospital and \\ ${ }^{3}$ Department of Radiology, Auckland Hospital, Auckland, New Zealand
}

\begin{abstract}
Summary: It has been reported that bone density is increased in patients with treated hypoparathyroidism, though it is unclear whether this increase is attributable to the condition itself or to its treatment. We have recently investigated a 70 year old woman with untreated hypoparathyroidism from the time of thyroid surgery at the age of 29 years. Bone mineral content of the non-dominant distal forearm was 3.7 standard deviations above the mean normal value found in 23 healthy volunteers of comparable menopausal age, and was also above the mean normal value found in premenopausal women. The vertebral mineral density of her lumbar spine (measured by quantitative computed tomography) was 3.0 standard deviations above the mean normal value. These findings suggest that high bone density is a feature of hypoparathyroidism per se and that postmenopausal bone loss may be significantly attenuated in this condition.
\end{abstract}

\section{Introduction}

Osteoporosis is a major cause of morbidity amongst women in Western societies. The advent of precise techniques for measuring bone density has allowed some insight into the hormonal and other factors which regulate bone mass. In women, one of the most important of these factors is oestrogen, but despite the recent identification of oestrogen receptors in bone, the mechanism by which it regulates bone metabolism remains uncertain. The possibility that oestrogen modifies skeletal sensitivity to parathyroid hormone has been suggested for many years. We have recently investigated a patient who developed post-surgical hypoparathyroidism premenopausally and had no specific treatment for this over the following 40 years. Her very high levels of bone density at the end of this time afford some insight into the interaction of oestrogen and parathyroid hormone in the regulation of bone mass.

\section{Case report}

A 70 year old Caucasian woman was referred to us after routine biochemical testing revealed a markedly reduced adjusted serum calcium concentration $(1.73 \mathrm{mmol} / \mathrm{l})$. She did not recall any previous estimation of her serum calcium having been

Correspondence: I.R. Reid, B.Sc., M.B., Ch.B., F.R.A.C.P. Accepted: 31 May 1990 carried out, and had had no blood tests at all for many years. On enquiry, she admitted to paraesthesiae in her fingers, muscle cramps especially in the right leg and frequent headaches. These symptoms had been present for many years. She was otherwise asymptomatic.

In the past, a partial thyroidectomy had been performed in 1946 for a benign goitre. She subsequently developed a change in voice and had intermittent dyspnoea culminating in the formation of a tracheostomy in 1958, at the time of an intercurrent respiratory tract infection. This produced lasting relief of her respiratory symptoms. She had been well since that time, apart from the development of a coarse tremor of the hands 5 years prior to the present referral (which responded well to an $l$-dopa preparation) and the diagnosis of hypertension one year later. She reached the menopause at age 41 . There was no family history of note. She had a well-balanced diet with a calcium content of approximately $1 \mathrm{~g} /$ day. Her medications on referral were $l$-dopa $100 \mathrm{mg} /$ carbidopa $25 \mathrm{mg}$ 4 times a day, quinine sulphate $300 \mathrm{mg} /$ day and enalapril $10 \mathrm{mg} / \mathrm{day}$.

On examination, she was clinically euthyroid with a coarse resting tremor of both hands but facial expression, muscle tone, ability to perform rapid alternating movements and gait were normal. No cataracts were detected. Her blood pressure was $180 / 120 \mathrm{mmHg}$, Chvostek's sign was absent but Trousseau's sign was present after 30 seconds. 
She weighed $71 \mathrm{~kg}$ and was $166 \mathrm{~cm}$ in height.

Biochemical testing confirmed euthyroidism and biochemical indices of calcium metabolism are set out in Table $I$. These show the changes of hypoparathyroidism - low total and ionized serum calcium levels with an inappropriately normal serum parathyroid hormone (PTH) concentration, high serum phosphate and increased tubular reabsorption of phosphate. Serum urea and creatinine levels were normal.

Bone mineral content (BMC) of the non-dominant distal forearm was measured using a Novo GT35 single photon absorptiometer, as previously described. ${ }^{1}$ The patient's value of 46 arbitrary units was 3.7 standard deviations above the mean value found in 23 healthy volunteers of comparable menopausal age (Figure 1). It was also above the mean BMC of premenopausal women (42 units). Vertebral mineral density (VMD) of the first three lumbar vertebrae was measured by quantitative computed tomography using a Philips Tomoscan 310 operating at $120 \mathrm{kVp}$ and a Cann-Genant phantom. The patient's VMD of $102 \mathrm{mg} / \mathrm{cm}^{3}$ was 3.0 standard deviations higher than the mean value found in 12 healthy volunteers matched for years since menopause (Figure 2).

\section{Discussion}

The biochemical findings at the time of the current assessment clearly support the diagnosis of hypoparathyroidism. The fact that the parathyroid hormone level was within the laboratory reference range does not argue against this diagnosis, since a normal level of PTH is clearly inappropriate in the face of this patient's hypocalcaemia. The PTH level is consistent with only partial loss of parathyroid function. The history of complicated thyroid surgery in 1946 dates the probable time of onset of her hypoparathyroidism which extended for 42 years, 30 of them postmenopausal, before diagnosis. The fact that the patient remained untreated through-

Table I Biochemical indices of calcium metabolism at presentation

\begin{tabular}{lcc}
\hline & Patient & $\begin{array}{c}\text { Reference } \\
\text { range }\end{array}$ \\
\hline Serum total calcium (mmol/l) & 1.94 & $2.2-2.6$ \\
Serum albumin $(\mathrm{g} / \mathrm{l})$ & 42 & $35-47$ \\
Serum ionized calcium (mmol/l) & 0.98 & $1.18-1.28$ \\
Serum phosphate (mmol/l) & 1.61 & $0.7-1.5$ \\
TmP/GFR* (mmol/l) & 1.66 & $0.8-1.4$ \\
Serum parathyroid hormone & 22 & $5-31$ \\
(pmol/l) & & \\
\hline
\end{tabular}

*Tubular maximum for the reabsorption of phosphate; $\dagger$ Radioimmunoassay with mid-molecule specificity.

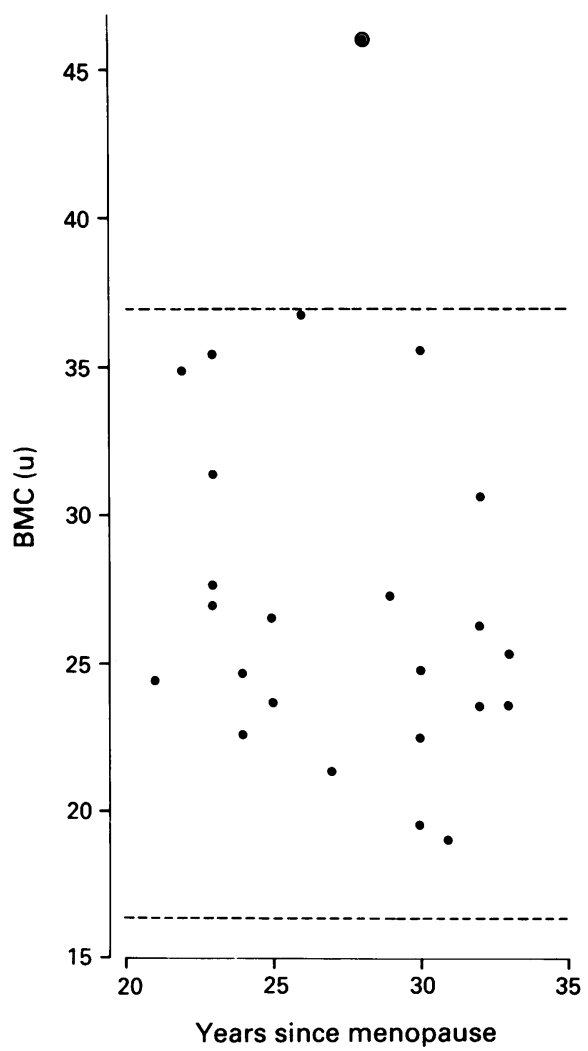

Figure 1 Bone mineral content (BMC) of the distal, non-dominant forearm measured by a Novo GT35 single photon absorptiometer in the patient (circled value) and in 23 healthy volunteers of comparable menopausal age. The data have been plotted as a function of menopausal age and the horizontal broken lines indicate mean \pm 2 s.d. for the normal subjects. BMC values are in arbitrary units.

out this period provides a unique opportunity to assess the effect of parathyroid hormone deficiency on postmenopausal bone loss. Our conclusions must be inferential since we have no earlier measurements of bone density in this patient. However, the finding of a forearm bone mineral content which is above the premenopausal mean value in a woman 30 years after the menopause, very strongly suggests that hypoparathyroidism greatly diminishes the rate of bone loss occurring in oestrogen-deficient women. The comparison of our patient's vertebral mineral density with that of control subjects suggests that a similar protective effect of hypoparathyroidism is operative in the spine. The normal premenopausal VMD on this model scanner is not available for comparison, however, VMD values measured on the Philips Tomoscan tend to be lower than those from other commonly used scanners. ${ }^{2}$ 


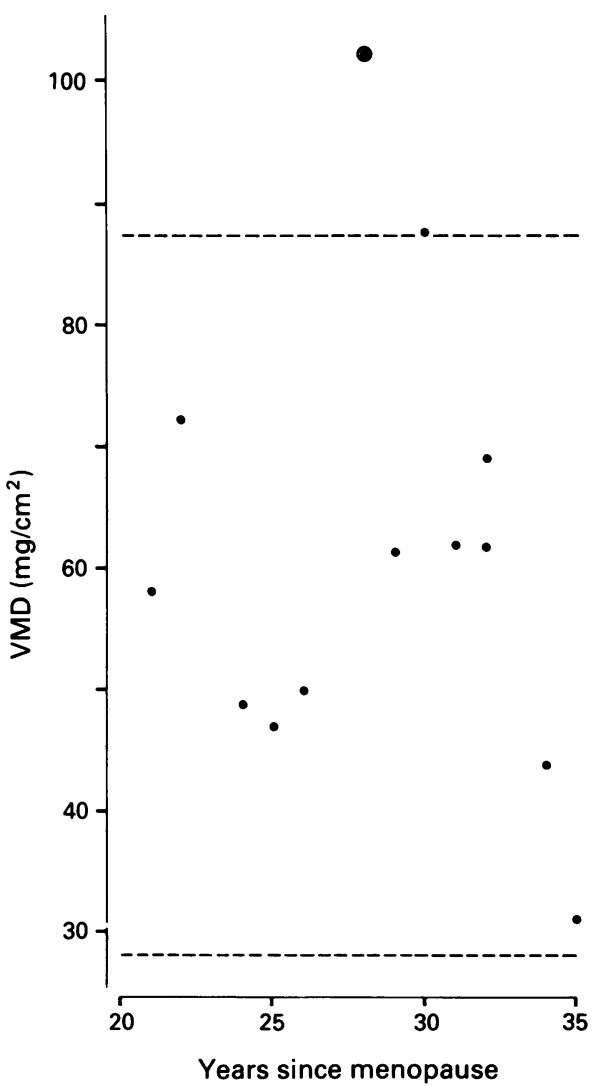

Figure 2 Vertebral mineral density (VMD) measured by a quantitative computed tomography using a Philips Tomsocan scanner. The patient's value has been circled and the other data points are derived from 12 healthy volunteers of comparable menopausal age. The horizontal broken lines indicate the mean \pm 2 s.d. for the normal subjects.

There are a small number of other reports of increased bone density in subjects with hypoparathyroidism. Dimich et al. ${ }^{3}$ commented on a subjective increase in radiographic bone density in some patients with hypoparathyroidism. Hossain et al. ${ }^{4}$ quantified this in the second metacarpal of hypo- parathyroid women. Premenopausally they found no difference in the metacarpal cortical areas of hypo- and hyperparathyroid women, but in postmenopausal subjects with hypoparathyroidism no postmenopausal bone loss was demonstrable. Hurxthal et al. ${ }^{5}$ documented increased spinal bone density using a semi-quantitative radiographic technique in a mixed group of hypoparathyroid patients. Similar results have been found in the metacarpals ${ }^{6}$ and in the lumbar spine using dual photon absorptiometry. ${ }^{7}$ Virtually all of the patients in these reports had received treatment with calcium supplements and vitamin D or its metabolites, usually over a period of many years. Significant changes in intestinal calcium absorption and in bone turnover would have been produced by these interventions and some uncertainty must remain regarding the relative contributions of hypoparathyroidism and its treatment to these increased bone densities.

The uniqueness of the present case is that such a substantial period of time passed from the development of parathyroid hormone deficiency until the time of institution of treatment (following the assessments reported herein). This significantly adds to the previously published evidence associating hypoparathyroidism with increased bone density and, in particular, supports the data of Hossain et al $^{4}{ }^{\text {suggesting that postmenopausal }}$ bone loss is greatly diminished in the presence of low levels of parathyroid hormone. Such a finding may prove to be of more than theoretical significance since drugs are now being developed which allow direct manipulation of parathyroid function. ${ }^{8}$ If it is confirmed that a state of partial hypoparathyroidism is associated with diminished postmenopausal bone loss, then these agents may find a role in the prevention or treatment of postmenopausal osteoporosis.

\section{Acknowledgements}

This work was supported by the Medical Research Council of New Zealand. The authors are grateful to Kathleen Bos for preparing the manuscript.

\section{References}

1. Reid, I.R., Mackie, M. \& Ibbertson, H.K. Bone mineral content in Polynesian and white New Zealand women. Br Med $J$ 1986, 292: 1547-1548.

2. Cann, C.E. Quantitative CT applications: Comparison of current scanners. Radiology 1987, 162: 257-261.

3. Dimich, A., Bedrossian, P.B. \& Wallach, S. Hypoparathyroidism. Clinical observations in 34 patients. Arch Intern Med 1967, 120: 449-458.

4. Hossain, M., Smith, D.A. \& Nordin, B.E.C. Parathyroid activity and post-menopausal osteoporosis. Lancet 1970, i: 809-811.

5. Hurxthal, L.M., Dotter, W.E., Vose, G.P. \& Sprinkle, E.E. Effect of postoperative hypoparathyroidism on bone density. Texas Rep Biol Med 1976, 34: 257-265.

6. Parfitt, A.M. Metacarpal cortical dimensions in hypoparathyroidism, primary hyperparathyroidism and chronic renal failure. Calcif Tissue Res 1977, 22 (Suppl): 329-331.

7. Seeman, E., Wahner, H.W., Offord, K.P., Kumar, R., Johnson, W.J. \& Riggs, B.L. Differential effects of endocrine dysfunction on the axial and the appendicular skeleton. $J$ Clin Invest 1982, 69: 1302-1309.

8. Brown, A.J., Ritter, C.R., Finch, J.L. et al. The noncalcemic analogue of vitamin $D, 22-o x a c a l c i t r i o l$, suppresses parathyroid hormone synthesis and secretion. J Clin Invest 1989, 84: 728-732. 